\title{
CORRIGENDUM
}

\section{Cucurbitacin Q: a selective STAT3 activation inhibitor with potent antitumor activity}

\author{
J Sun, MA Blaskovich, R Jove, SK Livingston, D Coppola and SM Sebti
}

Oncogene (2008) 27, 1344; doi:10.1038/sj.onc.1211028

us that HPLC/MS and NMR analysis of NSC135075 was inconsistent with its identification as Cuc Q. After purification of the single major component from NSC135075, and spectroscopic analysis, it was identified

Correction to: Oncogene (2005) 24, 3236-3245; as the natural product withacnistin (Wit) ( $\mathrm{J} \mathrm{Org} \mathrm{Chem}$ doi:10.1038/sj.onc.1208470; published online 21 February 2005.

Following publication of the above article, the authors have identified errors, which they now address as follows:

In the article entitled 'Cucurbitacin Q: a selective STAT3 activation inhibitor with potent antitumor activity' (Sun et al. (2005) Oncogene 24: 3236-3245), we have shown that treatment of human cancer cells that contain persistently activated tyrosine phosphorylated STAT3 (P-STAT3) with National Cancer Institute (NCI) library compound NSC135075 resulted in suppression of P-STAT3 but not P-JAK2 levels. NCI records indicated that NSC135075 corresponded to cucurbitacin $\mathrm{Q}$ and therefore in our publication we referred to it as such. However, recently the NCI alerted (1969) 34, 3858-3866) (see Supplementary Information). The major component purified from NSC135075, Wit, was provided to us by the NCI. With this, we have shown that pure Wit is as effective as NSC135075 at suppressing P-STAT3 but not P-JAK2 levels, inhibiting proliferation and inducing apoptosis. Supporting information, including the structure of Wit, HPLC/MS, NMR and methods, is available online at the Oncogene website.

Additional experiments pertaining to the identity of the compound were performed by Paul Klausmeyer, Thomas G McCloud (affiliated with National Products Support Group, SAIC-Frederick Inc., NCI-Frederick, USA), Tamara L Meragelman and David J Newman (affiliated with Natural Products Branch, Developmental Therapeutics Program, Division of Cancer Treatment and Diagnosis, NCI-Frederick, USA).

Supplementary Information accompanies the paper on the Oncogene website (http://www.nature.com/onc).

\section{ERRATUM}

\section{Ski can negatively regulates macrophage differentiation through its interaction with PU.1}

N Ueki, L Zhang and MJ Hayman

Oncogene (2008) 27, 1344; doi:10.1038/sj.onc.1211002

Correction to: Oncogene (2008) 27, 300-307; doi:10.1038/sj.onc.1210654; published online 9 July 2007
Due to a typesetting error, the name of the third author was misspelt. The corrected author list is shown above. 